\title{
Vaccine-induced Th17 cells are established as resident memory cells in the lung and promote local $\lg$ A responses
}

\author{
D Christensen ${ }^{1}$, R Mortensen $^{1}$, I Rosenkrands ${ }^{1}$, J Dietrich ${ }^{1}$ and P Andersen ${ }^{1}$
}

The ability to mount accelerated and efficient mucosal immune responses is critically important to prevent the establishment of many infections. Secretion of immunoglobulin A (IgA) is a key component in this first line of defense, but the underlying cellular mechanisms are still not completely understood. We have evaluated different routes of immunization and examined the requirements for IgA induction in the airway mucosa. We demonstrate that subcutaneous priming with a recombinant antigen in a Thelper (Th)17-inducing adjuvant followed by airway boosting promotes high and sustained levels of IgA in the lungs. This response is associated with germinal center formation in the lung-draining lymph nodes. The lung IgA response is dependent on Th17 cells and absent if interleukin (IL)-17 is depleted or when priming with vaccines inducing only Th1 or Th2 responses. We used intravascular staining to demonstrate that $\lg A+B$ cells and chemokine receptor 6 (CCR6) + Th17 cells are recruited to the lung parenchyma after the airway booster immunization. Once recruited to the lung parenchyma, the Th17 cells transform into resident lymphocytes that persist in the lung tissue for at least 10 weeks. Here, they facilitate the accelerated recruitment of $T$ and $B$ cells resulting in an accelerated IgA recall response to a second airway booster immunization.

\section{INTRODUCTION}

Most pathogens use the external barriers of the airways, intestines, or genital tract as ports of entry, and the ability to mount an efficient mucosal immune response at these surfaces is critically important for protection against many pathogens. It is thus a high priority to understand the induction and maintenance of mucosal immune responses in order to generate efficient vaccines against mucosal pathogens.

It is known that mucosal and systemic immune systems are separated, and that lymphocyte priming in, e.g., the lungs will create strong tropism of those lymphocytes to the airways, whereas parenteral immunization induces systemic immunity. ${ }^{1-3}$ Immunoglobulin A (IgA) is a critically important effector molecule at the mucosal surface. To date, little is known about the regulators of class switching to IgA, although transforming growth factor $\beta$, has been shown to have a direct role. $^{4-7}$ It is also known that $\mathrm{T}$ helper (Th) 17 cells are more abundantly present at the mucosal surface of the intestines, compared with other T-cell subsets, and intestinal interleukin
(IL)-17 expression correlates with IgA secretion. ${ }^{5}$ Th17 cells are furthermore suggested to be precursors for the follicular helper $\mathrm{T}\left(\mathrm{T}_{\mathrm{FH}}\right)$ cells that induce IgA class switch in Peyer's patches. ${ }^{8}$ Less is known about the potential role of Th17 cells for IgA induction in the airways, though their involvment is suggested by their high prevalence in lung tissue during airway infections. ${ }^{9,10}$ It is known that intranasal administration of IL-17 upregulates epithelial polymeric immunoglobulin receptor (pIgR) expression and promotes influx of B cells and IgA into the lungs. ${ }^{11}$ However, there is no evidence to suggest that Th17 cells have a direct role for IgA class switching and generation of IgA-producing plasma cells in the airways. Whether Th17 cells must be primed in the airways or distal mucosa-associated lymphoid tissues to acquire tropism to this tissue as observed after epicutaneous sensitazion ${ }^{12}$ or if parenterally primed Th17 cells can be relocated and obtain tropism to the airways is an area of ongoing research.

In the present study, the aim was to examine the role of parenterally primed T cells for IgA induction in the airways. To

1Department of Infectious Disease Immunology, Statens Serum Institut, Copenhagen S, Denmark. Correspondence: D Christensen orP Andersen (den@ssi.dk or pa@ssi.dk) Received 1 July 2015; accepted 25 February 2016; published online 6 April 2016. doi:10.1038/mi.2016.28 
induce high IgA titers, we present a vaccination protocol based on parenteral priming with the Th1/Th17-inducing adjuvant CAF01 ${ }^{13,14}$ (Cationic Adjuvant Formulation no. 1), followed by an airway booster immuization. We find that the induction of IgA-producing B cells in the lungs correlates with rapid homing of systemically primed chemokine receptor 6 (CCR6)-expressing Th17 cells to the lung parenchyma and germinal center (GC) formation in the lung-draining lymph nodes (LNs). The Th17 cells are established as tissue resident cells that promote accelerated recall IgA responses. These findings challenge the current dogma that only local mucosal priming creates strong tropism of lymphocytes to the airways, which is of great relevance for the design of vaccines against airway pathogens and for the induction of local IgA at mucosal sites.

\section{RESULTS}

Increased IgA in the airways by parenteral priming and airway boosting

Our first aim was to establish an immunization protocol that provides a strong local IgA response in the airways. We therefore compared the ability of different prime-boost immunization strategies to induce lung IgA responses. Mice were immunized twice with CAF01 adjuvanted Group A streptococcal C5a peptidase (ScpA) by different combinations of subcutaneous (s.c.) and airway (intranasal, i.n.) administration routes. We then measured IgG1, IgG2a, IgA, and IgM antibodies in the lung homogenate supernatant and interferon (IFN) $-\gamma$, IL-5, and IL-17 production by restimulated lung cells at days $1,3,7,14,21,28$, and 70 , as well as IgA-producing plasma cells at day 14 after the booster immunization.

As depicted in Figures $\mathbf{1 a}, \mathbf{b}, \mathbf{e}, \mathbf{f}$, the s.c./i.n. and s.c./s.c. immunizations resulted in high levels of IgG1 and IgG2a responses in lungs and serum. I.n./i.n. immunization for comparison gave IgG1 and IgG2a responses, 10-100-fold below the responses observed with the other strategies. The s.c./i.n. immunization resulted in a significantly elevated IgA titer already from day 7 , which was maintained for at least 10 weeks in both lungs and serum (Figures 1c, g). IgA was only transiently detected in the i.n./i.n. group after 2 weeks, after which the levels gradually returned to background levels 100-fold below the s.c./i.n. group. IgA was not detected in the lungs and serum after s.c/s.c. administration (Figures 1c, g). IgA responses in bronchoalveolar lavage collected at day 14 correlated with those in lung homogenate supernatant $\left(\mathrm{r}^{2}=0.9278\right.$; linear regression, $n=18$; data not shown). IgM was only detected in low amounts at day 7 (Figures 1d, h). Utilizing area under the curve as a measure for antibody secretion over time showed that s.c./i.n. immunization gave markedly increased secretion of IgA compared with the other strategies (Supplementary Figure S1A online). Correlating with these findings, s.c./i.n. immunization promoted high levels of T cells in the lungs, secreting high levels of cytokines (Figures 1i-k). IFN- $\gamma$ and IL-5 levels were significantly elevated only at day 21 (Figures $\mathbf{1 i}, \mathbf{j}$ respectively), whereas the IL-17 level was significantly elevated from day 3 and onwards (Figure 1k). I.n./i.n. immunization induced a slightly elevated (non-significant) IL-17 response compared with s.c./s.c. immunization at day 21 only.

The amount of ScpA-specific IgA antibody-secreting cells (ASCs) in perfused lungs increased significantly after s.c./i.n immunization to reach $76 \pm 21 \mathrm{IgA}$ ASCs per million day 14 (Figure 11). I.n./i.n. and s.c./s.c. immunization gave IgA ASC levels in the lungs comparable with background level in naïve mice ( $<20$ IgA ASCs per million). As expected, no significant increase in IgA ASCs was observed in the spleen, for any of the groups ( $<20$ IgA ASCs per million). The total amount of IgA ASCs in the lungs did not increase, suggesting no recruitment of non-related IgA ASCs (Supplementary Figure S1D). We furthermore observed that the IgA induction was dependent on the adjuvant in the mucosal booster, because boosting with non-adjuvanted ScpA induced insignificant lung IgA responses (data not shown). In summary, only s.c./i.n. facilitated sustained IgA production over time and significantly enhanced local IgA ASC numbers. Elevated IgA responses thus correlated with elevated IL-17 responses in lungs as observed after s.c./i.n. immunization and to a lesser extent i.n./i.n. immunization.

To further study the influence of the priming vaccine on the induction of IgA in the lungs, we compared CAF01 priming with two different vaccine adjuvants known to promote markedly different T-cell profiles: aluminum hydroxide $\left(\mathrm{Al}(\mathrm{OH})_{3} \text {; Th2-promoting }\right)^{15}$ and $\mathrm{CpG}$ Oligodeoxynucleotide (CpG; Th1-promoting). ${ }^{16}$ CAF01 was used for the i.n. booster immunization in all groups. As illustrated in Figure 1m, priming with $\mathrm{Al}(\mathrm{OH})_{3}$ and $\mathrm{CpG}$ resulted in a significantly lower IgA response in the lungs after boosting, compared with CAF01. To correlate this with the immune profile, we stimulated lung and spleen cells with the vaccine antigen and analyzed the secretion of IFN- $\gamma$, IL-5, and IL-17 in culture supernatant (Figure 1n). IFN- $\gamma$ release from splenocytes was promoted by both CpG and CAF01 (Figure 1n, top left), whereas only CAF01 priming mediated IFN- $\gamma$ induction in the lungs (Figure 1n, top right). IL-17 induction in the lungs and spleen was found only with CAF01 priming (Figure 1n, bottom). $\mathrm{Al}(\mathrm{OH})_{3}$ gave very limited cytokine release, and only IL-5 reached a measureable level in the lungs (Figure 1n, middle right). Our data therefore suggest that the IgA promoted by the s.c./i.n. immunization by CAF01 is related to an IL-17 response induced by the priming immunization.

\section{Parenteral prime and airway boost localize IgA + B cells and Th17 cells in the lung parenchyma}

We next studied in detail the B- and T-cell recruitment that takes place in s.c. primed animals during the airway boost (s.c./ i.n.) as compared with animals receiving a s.c. boost (s.c./s.c.), the latter inducing very similar IgG and IgG2a levels but no IgA. To first examine whether IgA + B cells were localized in the lung parenchyma or in the lung vasculature, s.c./i.n. immunized mice were subjected to in vivo intravascular staining. This was conducted by injecting fluorescein isothiocyanate (FITC)labeled anti-CD45 monoclonal antibody (mAb) intravenously (iv.CD45) $3 \mathrm{~min}$ before killing of the mice, thereby staining all intravascular, but not parenchymal, lymphocytes with 

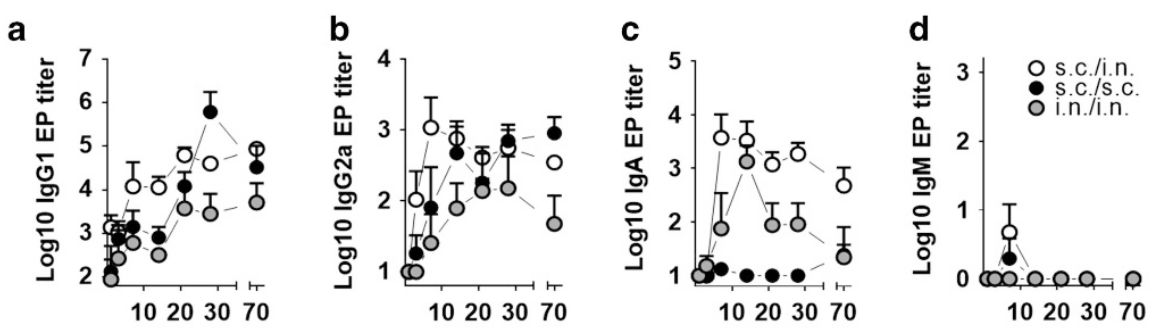

Lungs; days post last immunization
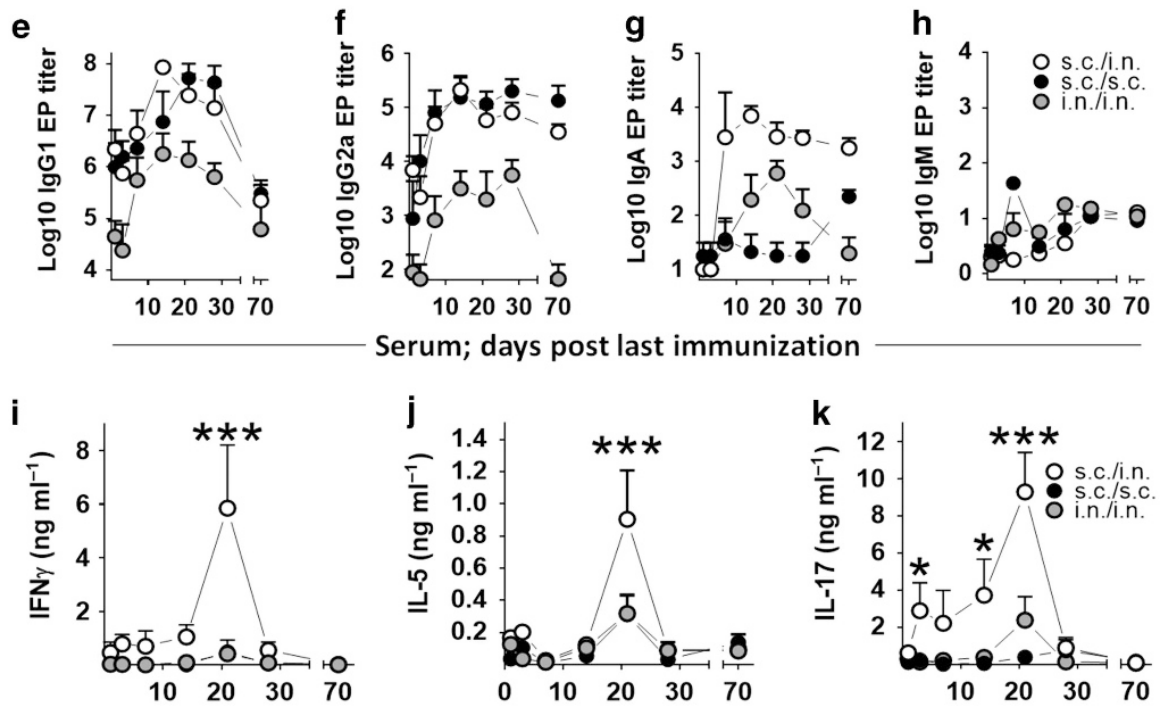

Serum; days post last immunization
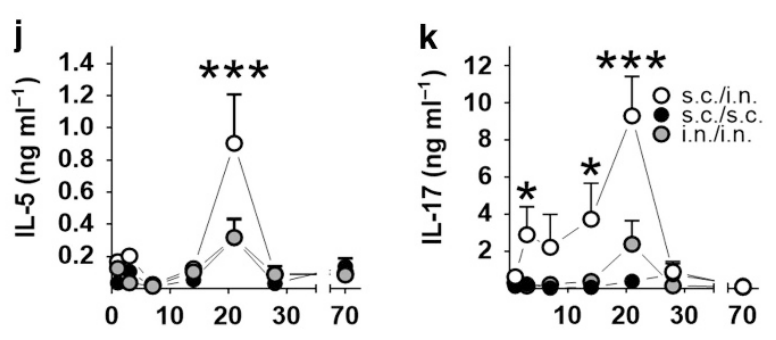

Lungs; days post last immunization
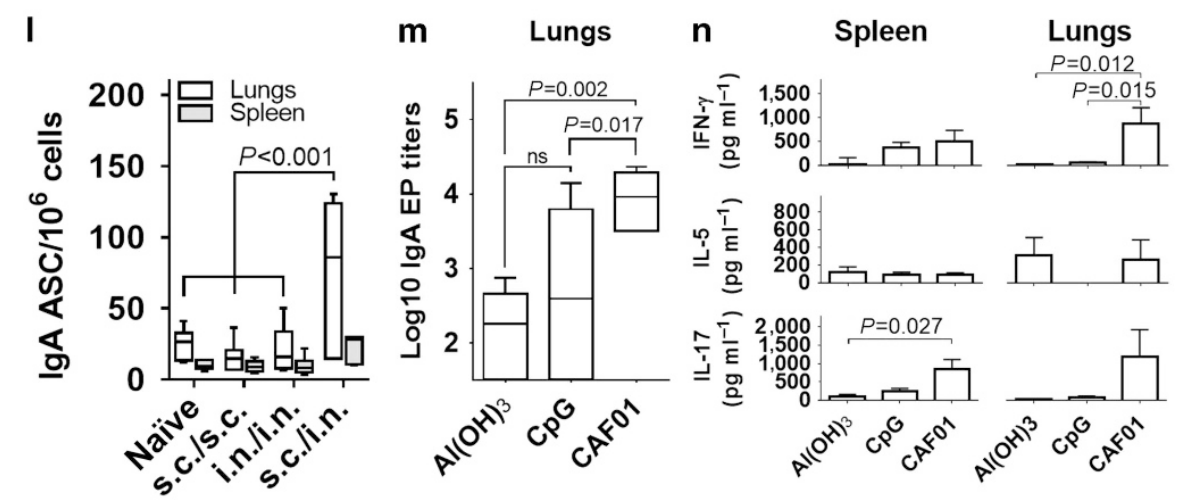

Figure 1 (a-k) Mice were immunized twice with 2 weeks interval by either the s.c./s.c. (๑), s.c./i.n. $(\bigcirc)$ or i.n./i.n. $(\bigcirc)$ regimes with ScpA adjuvanted with CAF01. IgG1, IgG2a, IgA, and IgM EP titers were determined in perfused homogenized lungs (a-d) and serum (e-h) at days 1, 3, 7, 14, 21, 28, and 70 after the booster immunization $(n=4)$. Cells from four individual mice immunized were isolated at days $1,3,7,14,21,28$, and 70 after booster immunization. Harvested cells were in vitro stimulated with ScpA for $72 \mathrm{~h}$ and IFN- $\gamma$, IL-5, and IL-17 levels were determined by MSD multiplex analysis (i-k). Statistical analysis: two-way ANOVA and Fishers Least Significant Difference post-test ${ }^{\star} P<0.05$, ${ }^{\star * *} P<0.001$. (I) ScpA-specific IgA ELISPOT on perfused lung and spleen cells 2 weeks after booster immunization. The number of ASCs determined in triplicates from six individual mice (mean w. s.e.m.). Statistical analysis: one-way ANOVA and Tukey's post-test. $(\mathbf{m}, \mathbf{n})$. Mice were primed with ScpA adjuvanted with aluminum hydroxide, CpG, or CAF01 and 2 weeks later boosted i.n. with ScpA/CAF01. (m) Boxplot ( $\mathrm{min} / \mathrm{max}$; median w. s.e.m.) showing lgA EP titers in lung supernatant, determined by ELISA 2 weeks after booster immunization. (n) Barplots (mean w. s.e.m.) showing IFN- $\gamma$, IL-5, and IL-17 levels in supernatant from ScpA-stimulated lung or spleen cells, determined by ELISA 2 weeks after booster immunization. Statistical analysis: one-way ANOVA and Tukey's post-test. ANOVA, analysis of variance; ASCs, antibody-secreting cells; ELISA, enzyme-linked immunosorbent assay; ELISPOT, enzyme-linked immunospot; EP, end point; IFN, interferon; IL, interleukin; i.n., intranasal; MSD, Meso Scale Discovery; s.c., subcutaneous; ScpA, streptococcal C5a peptidase.

FITC, as described by Anderson et al. ${ }^{17}$ Figure 2a depicts concatenation plots ('digital pooling', $n=6$ ) for iv.CD45 - and + populations of $\mathrm{CD} 19+\mathrm{B}$ cells, and their surface expression of IgA (flow cytometry, panel 1, Methods) 2 weeks after booster immunization. The percentage of $\operatorname{Ig} \mathrm{A}+\mathrm{CD} 19+\mathrm{B}$ cells in the vasculature (iv.CD45 + ) was not different between the s.c./s.c. and s.c./i.n. immunization groups ( 0.43 vs. $0.34 \%$ Figure 2 a) and did not differ from non-immunized controls. In 
contrast, the s.c./i.n. group showed a 10 -fold increase in $\operatorname{IgA}+\mathrm{B}$ cells in the lung parenchyma (Figures 2a, b). An elevated number of IgA + parenchymal B cells was also observed at day 42 after booster immunization in the s.c./i.n. group (data not shown).

Having demonstrated an increased lung recruitment of B cells in the s.c./i.n. immunized mice, we next focused on the $\mathrm{T}$ cells recruited to the lungs in s.c./i.n. vs. s.c./s.c. immunized mice. Again, cells in the parenchyma were distinguished from intravascular-localized cells. Lung cells were isolated at days 0 , 3,7 , and 14 after the booster immunization, stimulated in vitro with ScpA, and analyzed by intracellular cytokine staining (panel 2, Methods). On the basis of the observation that the lung IgA response correlated with IFN- $\gamma$ and IL-17 secretion induced by the priming vaccine, we assessed ScpA-specific $\mathrm{CD} 44+\mathrm{CD} 4+\mathrm{T}$ cells for their intracellular IFN- $\gamma$ or IL-17 cytokine expression (Figure 2c). The s.c./i.n. immunization strategy led to a markedly elevated infiltration of CD44+ $\mathrm{CD} 4+\mathrm{T}$ cells (Figure 2c; bottom) in the lungs, as compared with s.c./s.c. immunization (Figure 2c; top) that remained at the naïve background level (3.29 vs. $0.92 \%)$. The majority of these cells were localized in the lung parenchyma and the cytokine + iv.CD45 - cells reached 7\% (IFN- $\gamma+), 8.5 \%$ $(\mathrm{IL}-17+)$ of the CD44 + CD4 + T cells in the s.c./i.n. group, compared with less than $1 \%$ in the s.c./s.c. group (Figure 2c). Significantly increased levels of total CD $4+$ T cells (Figure 2d) and Ag-specific cytokine producing (cyt + ) $\mathrm{CD} 44+\mathrm{CD} 4+$ $\mathrm{T}$ cells (Figure 2e) in the lung parenchyma (iv.CD45 - ) were observed already at day 7 . When comparing the subpopulation distributions in the lungs at days $0,3,7$, and 14 in the s.c./i.n. group, it was evident that IL-17+CD44 + CD4 + T cells were recruited in an accelerated manner to the lung parenchyma compared with IFN- $\gamma+\mathrm{CD} 44+\mathrm{CD} 4+\mathrm{T}$ cells (Figure 2f). These IL-17- and IFN- $\gamma$-producing T cells were distinct populations (data not shown), as previously reported. ${ }^{14}$ ScpA-specific IL-17-producing T cells preceded IFN- $\gamma$-producing cells in the lung parenchyma already from day 3 and continued to outnumber IFN- $\gamma$-producing cells at later time points (Figure 2f), in agreement with the early IL-17 secretion from $\mathrm{T}$ cells isolated from the lung (Figure 1k). The IL-17+ population furthermore expressed higher levels of mucosal homing receptor CCR6 than both the IFN- $\gamma+$ and cyt $-\mathrm{CD} 44+\mathrm{CD} 4+\mathrm{T}$ cells (Figure $\mathbf{2 g}$ ).

We observed no ScpA-specific Th17 cells in the lung parenchyma and lung-draining LNs (tracheobronchial+ mediastinal, MLN + TLN) after s.c. priming, very low but detectable levels in the inguinal LN (ILNs) draining the s.c. injection site (fourfold increase over non-immunized), and elevated IL-17 production by lymphocytes from peripheral blood (581 \pm 307 vs. $36 \pm 16 \mathrm{pg} \mathrm{ml}^{-1}$ in non-immunized) (data not shown). Thus, the s.c./i.n. vaccine strategy led to increased localization of both $\operatorname{IgA}+\mathrm{B}$ cells and in particular Th17 cells in the lung parenchyma. As local secretion of IgA in the lungs of the s.c./i.n. immunized animals was closely associated with the early presence of IL-17-producing cells in the lungs after airway boost, we conducted a follow-up study where we compared the influx of IL-17-producing cells in the lung and lung-draining LNs (TLN + MLN) in s.c./i.n., i.n./i.n., and s.c./s.c. immunized animals. On day 14 post booster immunization, significantly elevated levels of IL-17+CD44+ $\mathrm{CD} 4+\mathrm{T}$ cells in lung parenchyma (iv.CD45 - ) were only observed after s.c./i.n. immunization although i.n./i.n. immunization also induced a slightly elevated (not statistically significant) response (Figure 2h). A similar pattern was observed in the lung-draining LNs (TLN + MLN; Figure 2i), whereas significant responses in the ILNs draining the s.c. immunization site were observed only after s.c./s.c. immunization (Figure 2j).

\section{Depletion of CD4 cells or IL-17 ablates lung IgA responses} Having shown a positive correlation between recruitment of Th17 cells and lung IgA, we next investigated whether Th17 cells were required for IgA induction. After the s.c. priming immunization, systemic IL-17 was depleted by i.p. injection of $100 \mu \mathrm{g}$ of anti-IL-17 mAb during booster immunization. As a positive control, $\mathrm{CD} 4+\mathrm{T}$ cells were depleted in a separate group. The experiment was terminated 10 days after boost, and homogenized perfused lungs were analyzed for humoral and cell-mediated immune responses. Intracellular cytokine staining (panel 2, Methods) of lung cells showed that IL-17 depletion reduced the proportion of activated CD $44+\mathrm{CD} 4+\mathrm{T}$ cells in the lungs from $4.99 \%$ in the isotype control group to $2.2 \%$ (Figure 3a left diagrams). CD4 depletion led to a complete removal of $\mathrm{CD} 4$ cells in the lungs. Within the activated $\mathrm{CD} 44+\mathrm{CD} 4+\mathrm{T}$ cells, there was also a reduction in both IL$17+$ (from 1.21 to $0.32 \%$ ) and IFN $\gamma+\mathrm{T}$ cells (from 0.8 to $0.01 \%$; Figure 3a). Both CD4 and IL-17 depletion led to a significant reduction in IgA titers in the lung (Figure $\mathbf{3 b}$ top; $\sim 2 \log$ reduction; $P<0.001$ ), whereas IgG was not significantly affected by the depletions (Figure $\mathbf{3} \mathbf{b}$ bottom). These data demonstrated that IL-17 is essential for vaccine-induced IgA, but not $\operatorname{IgG}$, responses in the airways.

\section{$T_{F H}$ cells and GC formation is not observed in the lungs but in lung-draining LNs}

Having confirmed that both $\mathrm{B}$ and $\mathrm{T}$ cells were recruited to the lung parenchyma during airway boost, we wanted to determine whether the $\mathrm{B}$ and $\mathrm{T}$ cells were localized in vaccine-induced tertiary lymphoid structures in the lung parenchyma or if GC formation was restricted to the draining LNs. We therefore investigated the induction of lymphoid GC B and $\mathrm{T}_{\mathrm{FH}}$ cells in lung tissue and TLN + MLN.

Mice were immunized s.c./i.n., i.n./i.n., or s.c./s.c. and injected with anti-CD45 $\mathrm{mAb}$ before they were killed. TLN + MLN, ILN, and lung cell suspensions were obtained on day 10 after booster immunization. Surface stainings were performed for detection of GC B cells (GL7 + CD95 + IgD$\mathrm{B} 220+\mathrm{CD} 19+$, panel 3, Methods, Figures 4a, b) and follicular T $\left(\mathrm{T}_{\mathrm{FH}}\right)$ cells $(\mathrm{CXCR} 5+\mathrm{PD} 1+\mathrm{CD} 44+\mathrm{CD} 4+$, panel 4 , Methods, Figures $4 c$, d). Both s.c./i.n. and s.c./s.c. immunization protocols gave increased GC B cells in the ILN draining the site of the priming immunization, whereas GC B cells in the TLN + MLN draining the lungs were found in high 


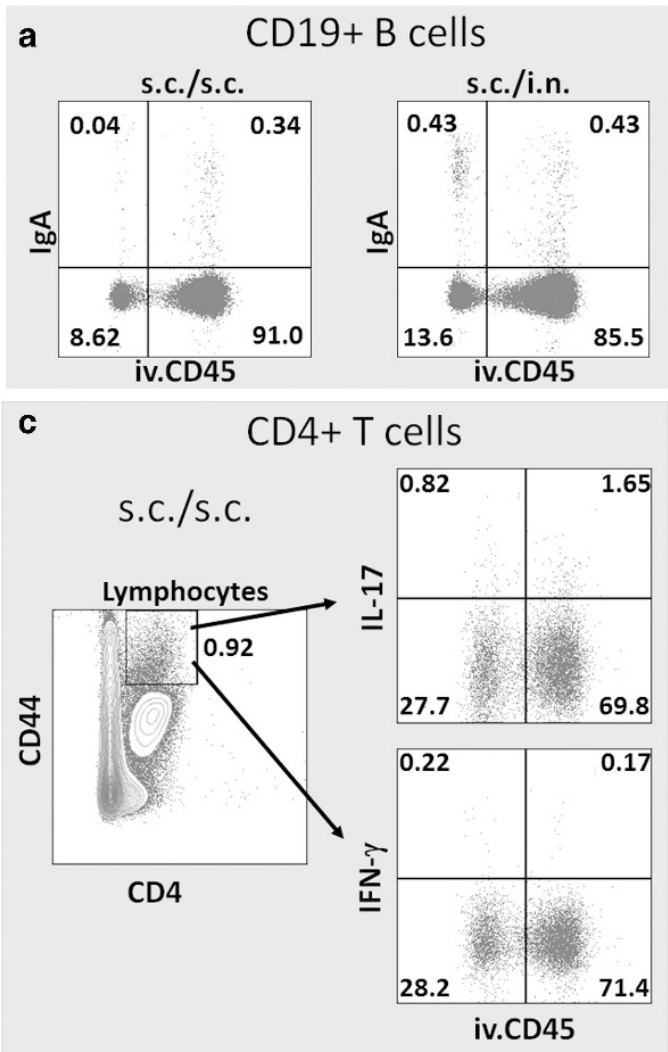

b

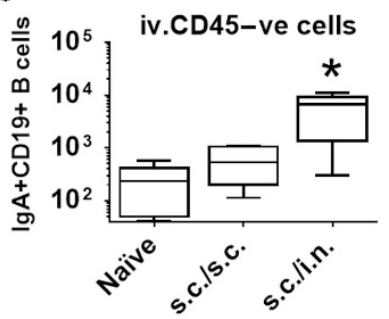

d
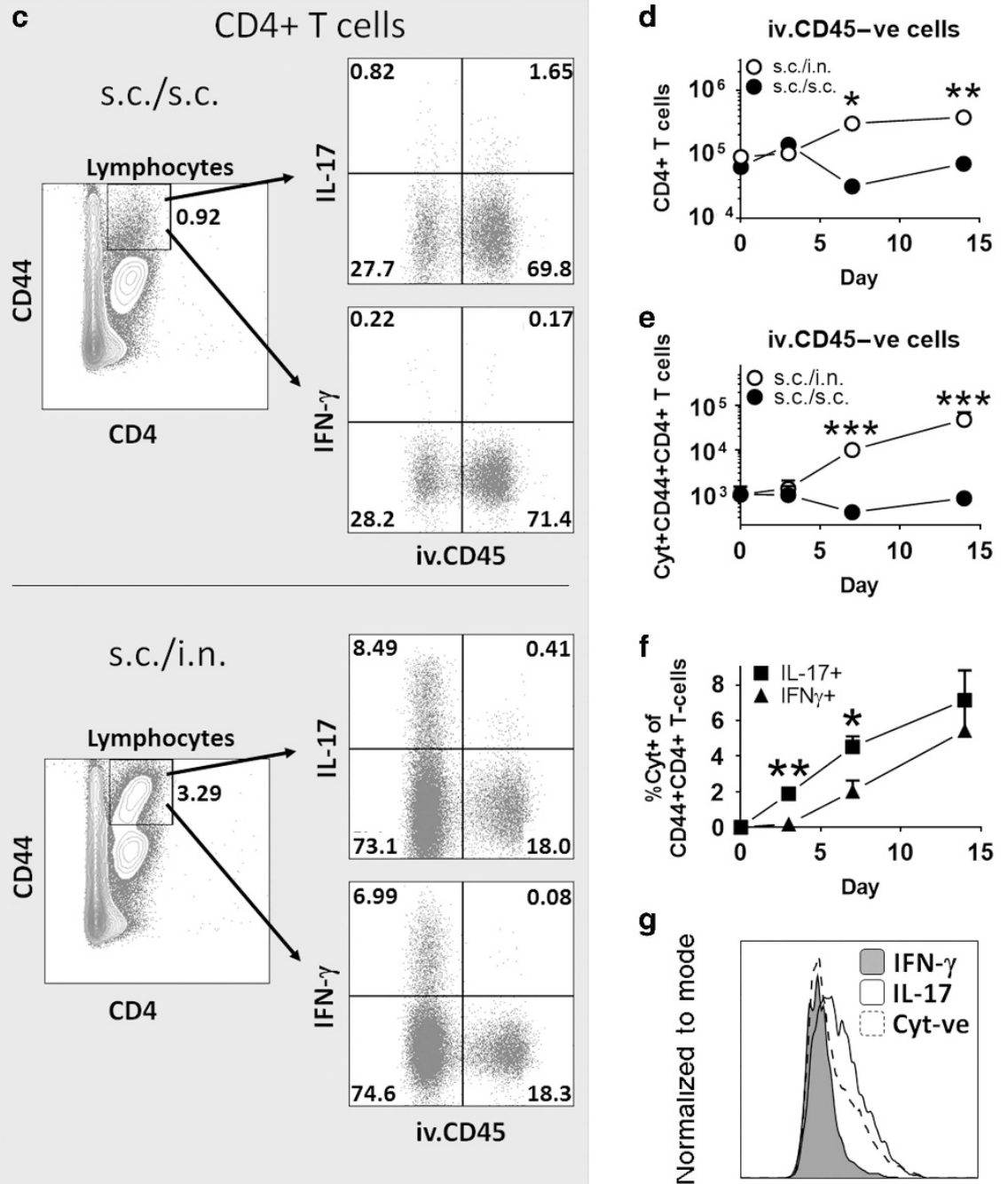

g
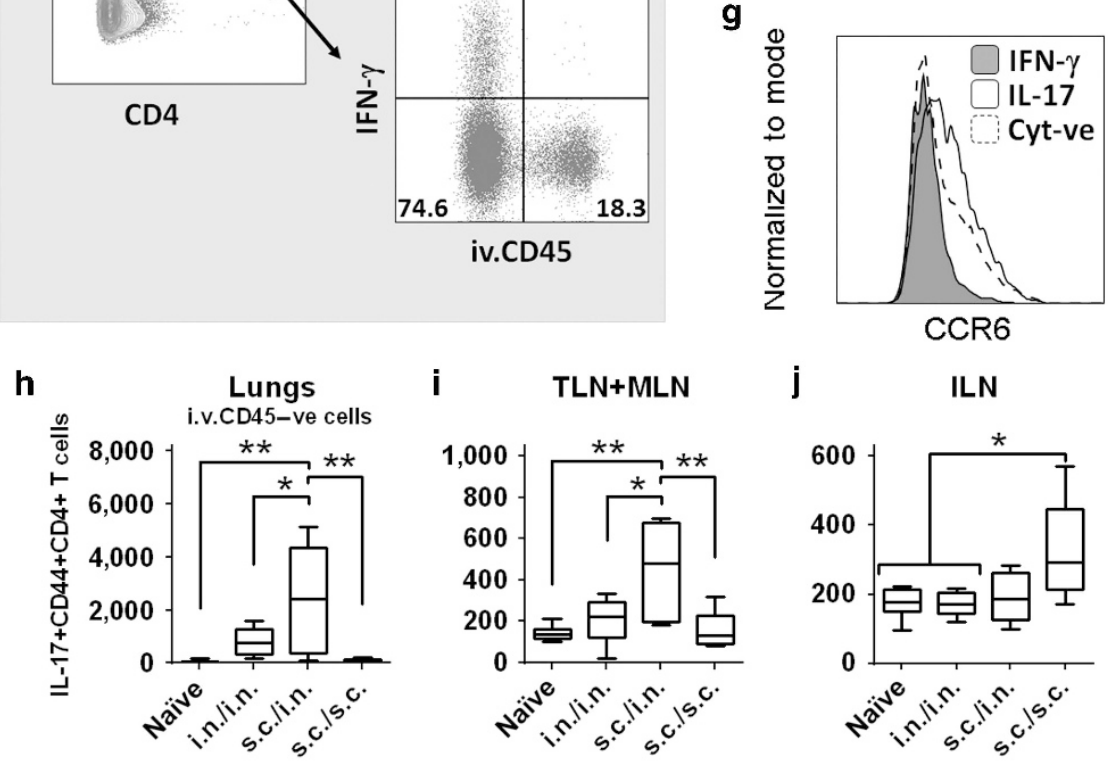
numbers after s.c./i.n. and i.n./i.n. immunization. None of the immunized animals showed evidence of GC B cells in the lung parenchyma (Figures $4 \mathbf{a}, \mathbf{b}$ ). Similarly only the s.c./i.n. and i.n./i.n. groups promoted $\mathrm{T}_{\mathrm{FH}}$ cells in the lungdraining LNs. Only i.n./i.n. immunized mice had low but detectable $\mathrm{T}_{\mathrm{FH}}$ cell levels in the lung parenchyma (Figures $4 \mathrm{c}$, d). In summary, these data suggest that airway boost (both after parenteral and mucosal prime) induces GC formation in the lung-draining LNs.

\section{Th17 cells persist as tissue-resident memory $\mathrm{T}$ cells responsible for accelerated IgA recall responses}

Having shown that the s.c./i.n. immunization strategy induced stronger IgA and IL-17+ T-cell responses in the lungs than i.n./i.n. and s.c./s.c. immunizations, we next investigated whether the Th17 cells represented a transient population of $\mathrm{T}$ cells or whether they persisted in the lungs as tissue-resident $\mathrm{T}$ cells. Mice received anti-CD45 mAb iv 6 weeks after the i.n. boost and lung cells were harvested. After stimulation with
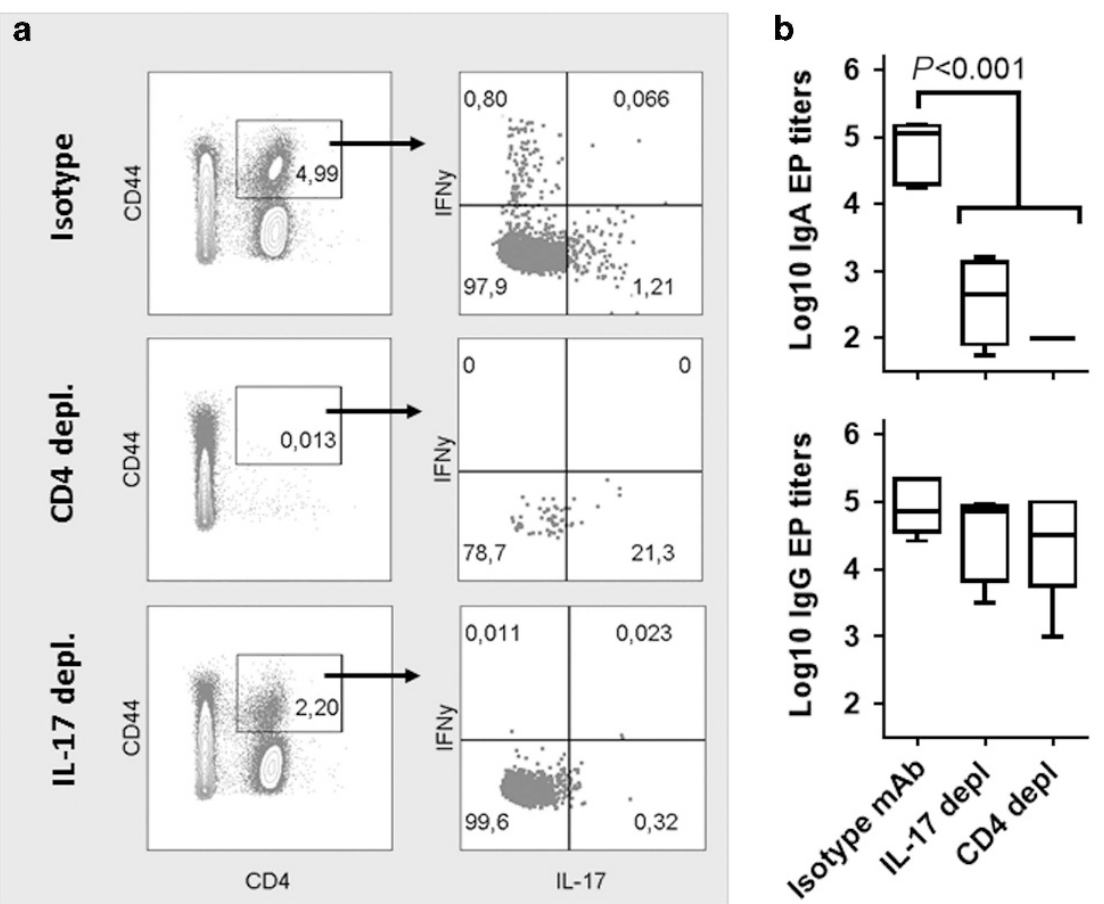

Figure 3 Mice were depleted for CD4 + T cells or IL-17. Mice received depleting antibodies starting 4 days before the intranasal booster immunization and continuing until 9 days after. The experiment was terminated 10 days after booster immunization. Ag-specific $T$ cells responses were assessed by IC Flow cytometry on in vitro ScpA-stimulated cells. (a) FACS plots of IL-17 and IFN- $\gamma$ positive cells in the CD $44+$ CD $4+$ T cell population in the lungs after depletion. (Concatenated from six individual animals). (b) Boxplots (min/max; median w. s.e.m.) showing IgA and IgG titers in homogenized perfused lungs, determined by ELISA. Statistical analysis: one-way ANOVA using Tukey's post-test. ANOVA, analysis of variance; ELISA, enzyme-linked immunosorbent assay; FACS, fluorescence-activated cell sorting; IC, intra cellular; IFN, interferon; IL, interleukin; ScpA, streptococcal C5a peptidase.

Figure 2 (a, b) Flow cytometry analysis was conducted to determine the location of CD19+ B cells after s.c./i.n. and s.c./s.c. immunization with ScpA/ CAF01 $(n=6)$. Lung cells were examined for labeling with iv injected FITC-conjugated anti-CD45.2 mAb 14 and 42 days post immunization. (a) The $\mathrm{CD} 19+\mathrm{B}$-cell staining is indicated. Dot plot shows IgA expression in iv.CD45 + (intravascular) and iv.CD45 - (parenchymal) cells. Figure shows concatenated data within groups ('digital pooling') from day 14. (b) Boxplot ( $\mathrm{min} / \mathrm{max}$; median w. s.e.m.) showing number of IgA + CD19+ B cells within the iv.CD45 - subset at day 14 after s.c./s.c. or s.c./i.n. immunization. Statistical analysis: one-way ANOVA and Tukey's post-test ${ }^{\star} P<0.05$. (c-f). IC Flow cytometric analysis of cytokine expression in CD4 T cells after s.c./i.n. and s.c./s.c. immunization with ScpA/CAF01. (c) Gating strategy for quantification and localization of lung-associated CD4 T cells in immunized mice (exemplified by day 14). Data are concatenated within groups. Lung cells were examined for labeling with iv-injected FITC-conjugated anti-CD $45.2 \mathrm{mAb} 0,3,7$, and 14 days post immunization by IC Flow cytometry analysis after stimulation with ScpA. Dot plot shows IL-17 or IFN- $\gamma$ expression in iv.CD45 + (intravascular) and iv.CD45 - (parenchymal) cells. (d) Number of CD4 + T cells in s.c./s.c. $(\bullet)$ and s.c./i.n. $(\bigcirc)$ immunized mice $(n=8)$. Statistical analysis: unpaired T tests at each individual time point ${ }^{\star} P<0.05$, ${ }^{\star \star} P<0.01$. (e) Number of cyt + CD44 + CD4 + T cells (any cytokine) in s.c./s.c. $(\bullet)$ and s.c./i.n. ( $O)$ immunized mice $(n=8)$. Statistical analysis: unpaired T tests at each individual time point ${ }^{* *} P<0.001$. (f) $\% \mathrm{IL}-17$ - or IFN- $\gamma$-expressing cells in the iv.CD45 - CD44 + CD4 + T cell population, in s.c./i.n. immunized mice. Statistical analysis: unpaired T tests at each individual time point ${ }^{\star} P<0.05$, ${ }^{\star \star} P<0.01$. (g) Histogram depicting expression of CCR6 on Cyt-iv.CD45CD44 + CD4 + T cells (dashed) IL17 + iv.CD45-CD44 + CD4 + T cells (transparent) and IFN- $\gamma+$ iv.CD45-CD44 + CD4 + T cells (dark) after s.c./i.n. immunization (exemplified by day 14, data concatenated within groups). (h-j) Number of IL-17+CD44+CD4+ T cells in i.n./i.n., s.c./i.n., and s.c./s.c. immunized mice $(n=6)$ in lung parenchyma (iv.CD45 - ; h), lung draining tracheobronchial and mediastinal LNs (TLN + MLN; i) and parenteral immunization draining ILN (j) 14 days post booster immunization. Statistical analysis: one-way ANOVA and Tukey's post-test ${ }^{\star} P<0.05,{ }^{* \star} P<0.01$. ANOVA, analysis of variance; FITC, fluorescein isothiocyanate; IC, intra cellular; IFN, interferon; IL, interleukin; ILN, inguinal lymph node; i.n., intranasal; iv, intravenously; LN, lymph node; mAb, monoclonal antibody; MLN, mediastinal lymph node; s.c., subcutaneous; ScpA, streptococcal C5a peptidase; TLN, tracheobronchial lymph nodes. 


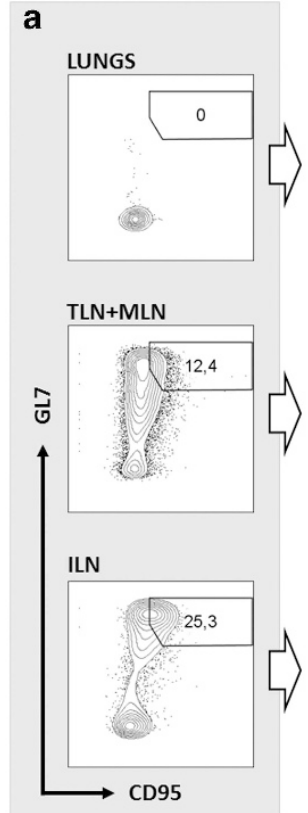

b
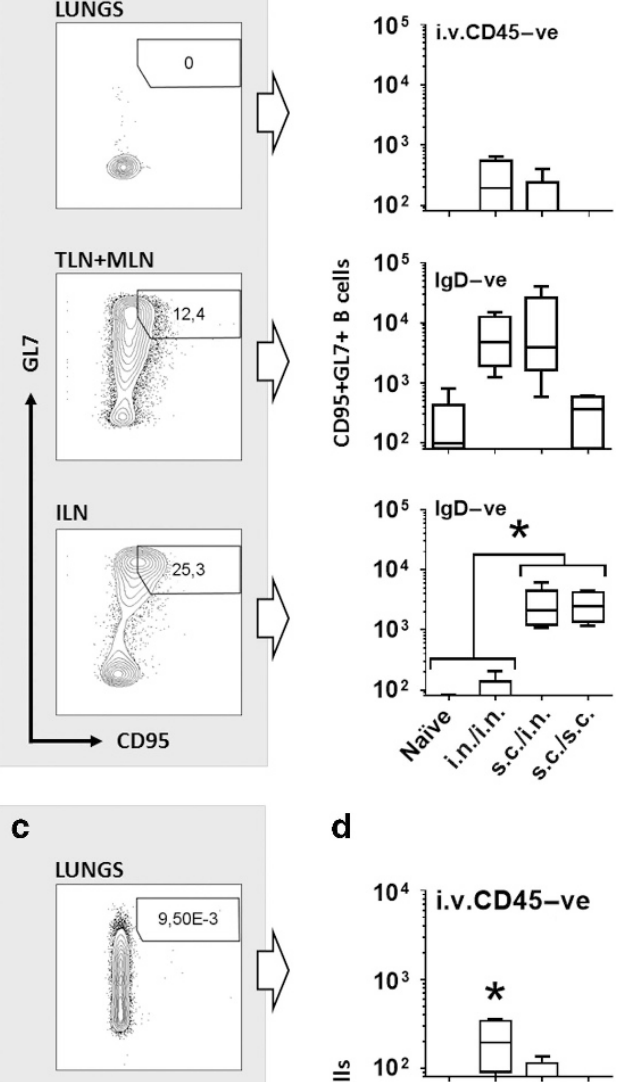

d
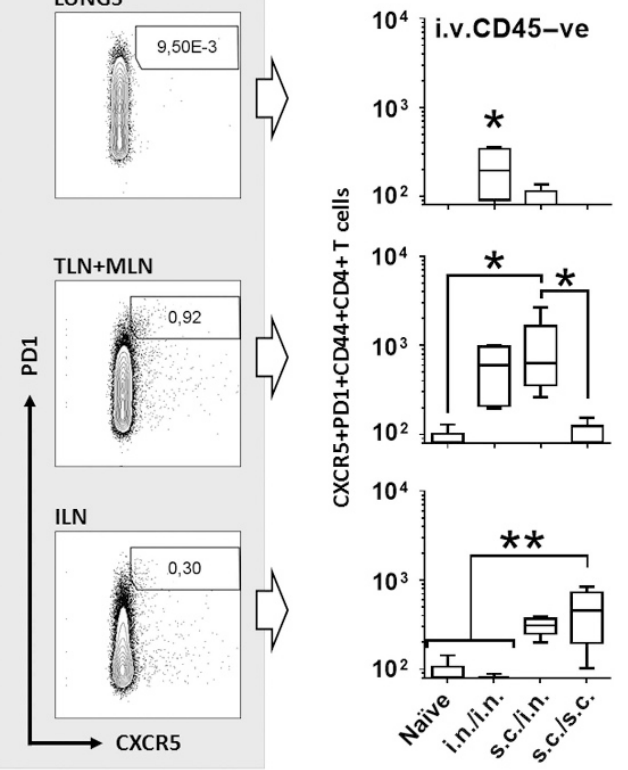

Figure 4 Flow cytometric analysis of GC B-cell and $\mathrm{T}_{\mathrm{FH}^{-}}$-cell formation in Lungs. TLN + MLN and ILN were analyzed 10 days after s.c./i.n., i.n./i.n. and s.c./s.c. immunization with ScpA/CAF01 compared with naïve background levels. Mice were intravascular stained by iv injection of FITC conjugated anti-CD45, 3 min before being killed. (a) Dot plots (concatenated within groups) showing GL7 vs. CD95 expression on $\mathrm{B} 220+\mathrm{CD} 19+\mathrm{B}$ cells from lungs (gated for iv.CD45 - ) or lymph nodes (LNs; gated for lgD - ). (b) Boxplots (min/max; median w. s.e.m.) showing total numbers of GC B cells in lungs (top), TLN + MLN (middle) and ILN (bottom). (c) Dot plot showing PD1 vs. CXCR5 expression on

CD44 + CD4 + T cells from lungs (gated for iv.CD45 - ) and lymph nodes. (d) Boxplots (min/max; median w. s.e.m.) showing total numbers of $\mathrm{T}_{\mathrm{FH}}$ cells in lungs (top), TLN + MLN (middle), and ILN (bottom). Statistical analysis: one-way ANOVA using Tukey's post-test ${ }^{*} P<0.05$.

ANOVA, analysis of variance; FITC, fluorescein isothiocyanate; GC, germinal center; ILN, inguinal lymph node; i.n., intranasal; iv, intravenously; MLN, mediastinal lymph node; s.c., subcutaneous; ScpA, streptococcal C5a peptidase; $T_{F H}$, follicular $T$ helper cells; TLN, tracheobronchial lymph nodes.
ScpA, the cells were stained for CD4, CD44, CD69, CD62L, and IL-17 (panel 5, Methods). At this late time point, mice still had a significant population of CD4 + CD44 + IL-17 + T cells (3.19\%) in their parenchyma (Figure 5a). More than half (66.4\%) of the IL-17+CD44 + CD4 $+\mathrm{T}$ cells in the lung parenchyma were CD69 + CD62L - tissue-resident memory $\mathrm{T}\left(\mathrm{T}_{\mathrm{RM}}\right)$ cells and the remaining were CD69-CD62L - effector memory $\mathrm{T}$ cells (Figure 5a). This distribution was markedly different from IL17 + T cells in the vasculature that contained only $17.2 \%$ CD69 + CD62 $-\mathrm{T}_{\mathrm{RM}}$ cells but a substantial proportion of CD69-CD62L + central memory $\mathrm{T}\left(\mathrm{T}_{\mathrm{CM}}\right)$ cells, not found in lung parenchyma (Figure 5a).

To investigate whether the $T_{\mathrm{RM}}$ cells had a role in a functional long-lived recall response, s.c./i.n., i.n./i.n. and s.c./s.c. immunized mice were given a second i.n. booster immunization 10 weeks after a completed immunization. A second boost resulted in 1-2 $\log$ higher IgA levels in the s.c./i.n. group compared with the i.n./i.n. and s.c/s.c. groups at all time points (Figure 5b). Notably, the IgA levels in the mice from the s.c./i.n. group had significantly elevated ScpA-specific IgA at the time 0 of this experiment, i.e., at the time of the second booster immunization (Figure 5b). Although i.n./i.n. immunization gave a substantial IgA response after the initial immunization (Figure 1c), this protocol failed to induce IgA following late i.n. boost. For comparison, elevated IgA levels were detected in the s.c./s.c. group, but only 2 weeks after late i.n. boost (Figure 5b). IL-17 induction was also highly accelerated and significantly higher in the s.c./i.n. group as compared with both s.c./s.c. and i.n./i.n. immunization (Figure 5c). This suggests that IgA-secreting B cells and IL-17-producing $\mathrm{T}$ cells remain in the lung parenchyma after s.c./i.n. immunization.

Finally, we evaluated the relative role of tissue resident and newly recruited $\mathrm{T}$ and $\mathrm{B}$ cells from the $\mathrm{LN}$ in the accelerated recall response observed in the s.c./i.n. group. This was addressed by blocking cell exit from the LNs by treatment with fingolimod (FTY720) during a second i.n. booster immunization. Mice immunized s.c./i.n. were rested for 10 weeks. Thereafter, half of the animals received $70 \mu \mathrm{g}$ fingolimod in $200 \mu \mathrm{l}$ water orally per day from 2 days before and until 2 weeks after the i.n. boost. Before killing the mice, they received labeled anti-CD45 $\mathrm{mAb}$ iv and lungs were analyzed for IgA and Th17 cells. Both Th17 cells (Figure 5d) and secreted IgA levels (Figure 5e) were significantly elevated by the i.n. boost, but fingolimod treatment almost completely abolished this booster effect. Only a minimal non-significant IgA increase was found in the fingolimod-treated animals. This suggests that the main role for tissue-resident Th17 cells in the lung parenchyma is to facilitate an accelerated recruitment of new $B$ and $T$ cells responsible for the rapidly emerging immunity in the lungs.

\section{DISCUSSION}

Little is known about the role of Th17 cells in the induction of IgA in the airways. Recent studies have demonstrated a Th17mediated upregulation of $\mathrm{pIgR}$ expression by the epithelium accompanied by influx of B cells and transport of secretory IgA and IgM into the airways. ${ }^{11,18}$ However, the role of Th17 cells in 


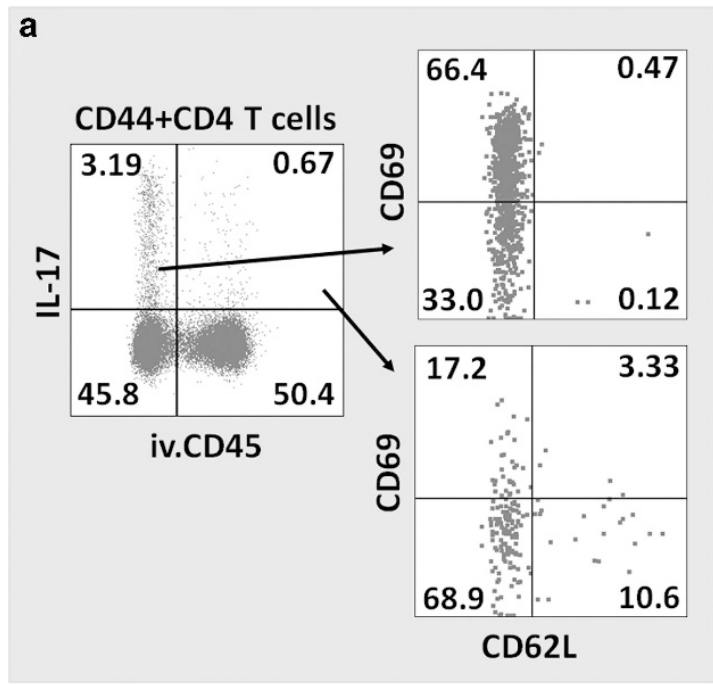

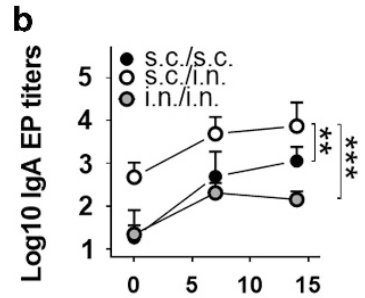

Days post second boost

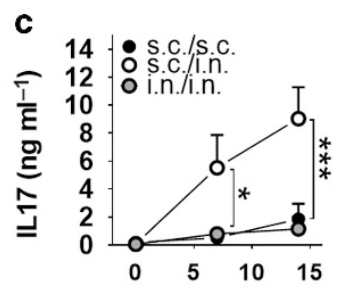

Days post second boost
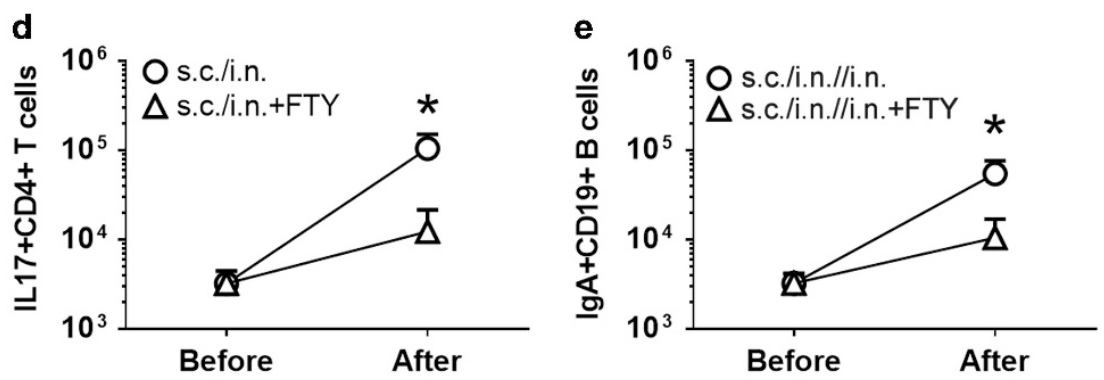

Figure 5 (a) IC Flow cytometry analysis of parenchymal T cells 42 days after s.c./i.n. immunization with ScpA/CAF01. Mice were intravascular stained by iv injection of FITC-conjugated iv.CD45, 3 min before being killed. CD44 + CD4 + T cell populations were subdivided according to their iv.CD45 staining and expression of IL-17. iv.CD45 - and iv.CD45 + IL17 + CD44 + CD4 + T cells were further subdivided according to their CD62L vs. CD69 expression. (b, c) Mice immunized either s.c./s.c. (•), s.c./i.n. ( $)$ ) or i.n./i.n. (○) received a second boost of ScpA/CAF01 administered i.n. 70 days later and mice were killed before and 7 and 14 days after the second booster immunization. (b) IgA end point titers were determined in supernatants of perfused and homogenized lungs from individual mice. The amount of secreted antibody was analyzed by ELISA. (c) Lung cells from individual mice $(n=4)$ were harvested and stimulated with ScpA for $72 \mathrm{~h}$ and the IL-17 levels determined by MSD analysis of culture supernatants. Statistical analysis: one-way ANOVA using Tukey's post-test. (d, e) In a similar study, half of the mice that received the second boost $(n=12)$ received FTY720 treatment by an oral stomach tube, from 1 day before until termination of the experiment day 7 after a second booster immunization. The mice were intravascular stained by iv injection of FITC-conjugated iv.CD45 and (d) Th17 cells and (e) IgA + B cells in the parenchyma were analyzed by IC flow cytometry. Statistical analysis: one-way ANOVA using Tukey's post-test. ${ }^{*} P<0.05$. ANOVA, analysis of variance; ELISA, enzyme-linked immunosorbent assay; FITC, fluorescein isothiocyanate; IC, intra cellular; IL, interleukin; i.n., intranasal; MSD, Meso Scale Discovery; s.c., subcutaneous; ScpA, streptococcal C5a peptidase.

establishment of local immunity and an IgA response in the airways is not clear, and most of what we know about the association between Th17 and IgA comes from studying the intestinal immunity.

In the present study, we introduce an optimized immunization protocol for induction of Th17 and IgA responses in the airways. We show for the first time that systemically induced Th17 cells are preferentially recruited to the lungs after airway exposure to the cognate $\mathrm{Ag}$, and that this is accompanied by a local IgA response. We also show that depletion of IL-17 abrogates the recruitment of $\mathrm{T}$ cells to the lungs and significantly reduces the local IgA response as well as the Th1 response (IFN- $\gamma+\mathrm{CD} 44+\mathrm{CD} 4+\mathrm{T}$ cells). The latter observation has been reported previously by Khader et al. ${ }^{19}$ showing that IL-17 depletion during Mycobacterium tuberculosis challenge reduced lung accumulation of IFN- $\gamma+\mathrm{CD} 4+$ $\mathrm{T}$ cells. In contrast, IgG responses were unaffected by IL-17 depletion. These results are in agreement with previous studies on the role of Th17 cells for intestinal IgA induction. Even though there is no indication that IL-17 has a direct role for the IgA isotype switch of B cells in the Peyer's patches, a number of publications have recently shown that Th17 cells are essential for this to occur. ${ }^{8,20,21}$ Hirota et al. ${ }^{8}$ recently showed that Th17 cells have the ability to induce GCs and transform into IL-21producing $\mathrm{T}_{\mathrm{FH}}$ cells. In line with this, Cao et al. ${ }^{21}$ showed that intestinal IgA was impaired in mice deficient in IL-17 or IL-21 signaling, but that only IL-21 is able to augment B-cell differentiation to IgA + cells. In addition, Th17 cells may also increase expression of the pIgR in the airway epithelium, thus leading to increased secretory levels of IgA as reported by Jaffar et al. ${ }^{11}$ Together with our data, these findings suggest that Th17 cells are important for both IgA isotype switch and local secretion of IgA not only in the gut, but also in the airway tissue.

It is well established that lymphocyte activation within a peripheral organ and/or its draining LNs will create tropism to that organ. However, our data suggest an ability of parenterally 
primed Th17 cells to home to mucosal tissues and establish themselves in these tissues as $\mathrm{T}_{\mathrm{RM}}$ cells. Thus, s.c./i.n. immunized mice mounted stronger Th17/IgA responses in the lungs than both s.c./s.c. and i.n./i.n. immunized animals.

Th17 cells are efficiently induced through mucosal infection or immunization ${ }^{22,23}$ and express tissue-selective homing receptors, such as CCR6. Indeed, we found that Th17 cells in the lungs expressed significantly higher levels of CCR6 than the Th1 cells, in support of previous observations, showing that CAF01-induced Th17 cells express higher levels of CCR6 than the Th1 subset. ${ }^{14}$ The ability of Th17 cells to establish a strong memory response is controversial. Studies in mice have shown that mucosally imprinted Th17 cells are short-lived, ${ }^{22}$ whereas studies in both primates and humans have detected Th17 memory cells after recovery from infection. ${ }^{24,25}$ Studies from our lab have previously shown that CAF01 induces a stable Th17 memory response by parenteral immunization in mice. ${ }^{14}$ In line with this, we show here that Th17 cells and IgA + B cells persist in lung parenchyma for at least 10 weeks after i.n. booster immunization, suggesting that Th17 cells can establish as $\mathrm{T}_{\mathrm{RM}}$ cells. Importantly, the presence of Th17 cells in the tissue facilitates accelerated recall responses with rapidly increasing IgA levels when the mice receive an i.n. booster immunization 10 weeks after s.c./i.n. immunization. Interestingly, this IgA recall response was not seen after late boost of i.n./i.n. immunization, although this protocol was associated with primary GC formation in the lung draining LNs and IgA production, suggesting that insufficient Th17 memory limits the ability to maintain a long-lived $\operatorname{IgA}$ recall response. Although most focus has been directed towards CD8+ $\mathrm{T}_{\mathrm{RM}}$ cells, ${ }^{26}$ recent data have demonstrated that $\mathrm{CD} 4+$ $\mathrm{T}$ cells reside in the tissue as well. For instance, respiratory memory CD $4+\mathrm{T}$ cells in the lungs of mice and humans have phenotypes clearly distinct from those found in lymphoid tissue or in blood. ${ }^{27}$ Moreover, influenza and M. tuberculosis lung infections induce CD4 + T cells with selective tropism towards the lungs. ${ }^{28,29}$ All these studies show that the $\mathrm{T}_{\mathrm{RM}}$ cells express CD69, whereas the systemic T cells do not. In addition, CD69 expression is also associated with $\mathrm{T}_{\mathrm{RM}}$ cells in the skin, ${ }^{30}$ genital tract, ${ }^{31}$ and bone marrow. ${ }^{32}$ Our data show that systemically primed Th17 cells establish CD62L-CD69 $+\mathrm{T}_{\mathrm{RM}}$ cells in the lungs, whereas CD62L + CD69 - $\mathrm{T}_{\mathrm{CM}}$ cells, classically found in lymphoid tissues, are absent (Figure 5a). We did not observe any $\mathrm{GC} B$ cells and $\mathrm{T}_{\mathrm{FH}}$ cells in the lungs after s.c./i.n. immunization, indicating that tertiary lymphoid structures are not formed.

In summary, our data show that vaccine-specific Th17 cells induced by parenteral immunization are recruited to the lung parenchyma after airway boost where they facilitate the accumulation of $\operatorname{IgA}+\mathrm{B}$ cells and rapidly increasing local IgA levels. This is associated with $\mathrm{T}_{\mathrm{FH}}$ and $\mathrm{GC}$ formation in the regional LNs. The Th17 cells establish $\mathrm{T}_{\mathrm{RM}}$ cells that remain in the lung tissue, resulting in long-term increased levels of secreted IgA. This local memory results in highly accelerated Band $\mathrm{T}$-cell recall responses in the lungs after another airway boost.
The role of Th17 cells in humans is not yet clear. However, there is increasing evidence supporting that Th17 cells have similar roles in humans and mice. ${ }^{33,34}$ Our data therefore suggest an immunization strategy for mucosal pathogens involving parenteral priming of a Th17 response followed by mucosal boosting. We demonstrate that this strategy results in the establishment of Th17 cells and high levels of local IgA in the airway. This strategy is particularly interesting, considering the need for safe vaccines with the ability to promote local immunity against infections of the lung, gut, and genital tract through a minimal number of mucosal immunizations. Recently published data from our laboratory have applied the CAF01 prime/intranasal boost strategy and demonstrated strong mucosal IgA responses to a chlamydia vaccine candidate in pigs. ${ }^{35}$ This vaccine strategy is currently being prepared for human clinical trials.

\section{METHODS}

Animals. Female CB6F1 hybrid mice (Harlan Scandinavia, Denmark) aged 6-8 weeks were kept at the experimental animal facilities at Statens Serum Institut and handled by authorized personnel. All manipulations were conducted in accordance with the regulations of the Danish Ministry of Justice and animal protection committees under permit 2009/561-1655 and in compliance with EC Directive 86/ 609.

Antigens, adjuvant, and immunizations. The vaccine protein ScpA, described previously by Cleary et al. ${ }^{36}$ (UniProt P15926), was recombinantly expressed in $E$. coli with an $\mathrm{N}$-terminal $\mathrm{His}_{6}$-tag, affinity-purified, subjected to ion exchange chromatography, and analyzed by mass spectrometry and sodium dodecyl sulfate-polyacrylamide gel electrophoresis, as previously described. ${ }^{37}$ CAF01 $(\mathrm{DDA} / \mathrm{TDB})^{38}$ was used as the adjuvant at a dose of $250 / 50 \mu \mathrm{g}$. Mice were immunized by the subcutaneous route (s.c., volume $200 \mu \mathrm{l}$ ) at the base of the tail or intranasal (i.n., volume $2 \times 20 \mu \mathrm{l}$ ). Two immunizations were given with 2 weeks interval.

In vivo staining with anti-CD45.2. Anti-CD45.2-fluorescein isothiocyanate (clone 104; BD Biosciences, Albertslund, Denmark) was diluted to $10 \mu \mathrm{g} \mathrm{ml}^{-1}$ in sterile phosphate-buffered saline (PBS); $250 \mu \mathrm{l}$ of the solution was injected iv via the tail vein, $3 \mathrm{~min}$ before killing of the mice.

FTY720 treatment. FTY720 (Sigma-Aldrich, Gillingham, UK) was diluted to $350 \mu \mathrm{g} \mathrm{ml}^{-1}$ in sterile water for injection. Mice received $200 \mu \mathrm{l}$ daily by stomach intubation from one day before until termination of the experiment.

Sample collection and cell preparation. Samples were collected from four to eight mice per group. Blood was obtained by submandibular bleeding. Lung cells were isolated by transferring lungs to gentleMACS C Tubes (Miltenyi Biotec GmbH, Bergisch Gladbach, Germany) containing $2 \mathrm{ml}$ RPMI 1640, 5\% fetal calf serum (FCS), and $0.8 \mathrm{mg} \mathrm{ml}^{-1}$ Collagenase type IV (Sigma-Adrich, St. Louis, MO) and dissociated into 1-2 $\mathrm{mm}$ pieces using the gentleMACS Octo dissociator (Miltenyi Biotec $\mathrm{GmbH}$ ), followed by $1 \mathrm{~h}$ incubation at $37^{\circ} \mathrm{C}$. The samples were returned to the gentleMACS and dissociated followed by centrifugation $(700 \times g, 5 \mathrm{~min})$. Supernatants were collected for antibody detection and the pellets were homogenized through cell-strainer (Falcon, Durham, NC) and washed twice in RPMI-1640 (Gibco Invitrogen, Hvidovre, Denmark). Cell cultures were performed in microtiter plates (Nunc, Roskilde, Denmark) containing $2 \times 10^{5}$ cells per well (Meso Scale Discovery) or $1 \times 10^{6}$ cells per well (fluorescence-activated cell sorting (FACS)/enzymelinked immunospot (ELISPOT)) in $100 \mu \mathrm{l}$ RPMI-1640 (Gibco 
Invitrogen) supplemented with $5 \times 10^{-5} \mathrm{M}$ 2-mercaptoethanol, $1 \mathrm{mM}$ glutamine, $1 \%$ pyruvate, $1 \%$ penicillin-streptomycin, $1 \%$ HEPES, and 10\% FCS (Gibco Invitrogen).

Antibody detection. Maxisorb Plates (Nunc) were coated with ScpA $\left(0.5 \mu \mathrm{g} \mathrm{ml}^{-1}\right)$. Serum and lung samples were fivefold serially diluted 8-12 times from a 1:1 dilution on the plates. IgA was detected with horse radish peroxidase (HRP)-conjugated goat anti-mouse IgA mAb (Invitrogen, Hvidovre, Denmark) diluted 1:5,000. IgG1 was detected with HRP-conjugated goat anti-mouse IgG1 mAb (Southern Biotech, Birmingham, AL) diluted 1:20,000. IgG2a was detected with HRPconjugated rabbit anti-mouse IgG2a mAb (Invitrogen) diluted 1:5,000. IgM was detected with HRP-conjugated goat anti-mouse IgM mAb (Southern Biotech) diluted 1:5,000. Substrate was 3,3,5,5-tetramethylbenzidine-PLUS (Kem-En-TEC, Taastrup, Denmark). Reciprocal serum dilutions corresponding to the end point titer (cutoff Optical Density $(\mathrm{OD})=0.1$ ) were computed using GraphPad Prism v6.04 (GraphPad Software Inc., La Jolla, CA).

ELISPOT assay. IgA ELISPOT was performed according to the manufacturer's instructions (MABTECH AB, Nacka Strand, Sweden). Polyvinylidene fluoride plates (Millipore, Hellerup, Denmark) were pretreated with ethanol for $1 \mathrm{~min}$ and washed $5 \times$ with sterile water. The plates were then coated with $100 \mu \mathrm{l}$ per well of ScpA solution $\left(5 \mu \mathrm{g} \mathrm{ml}^{-1}\right)$ and incubated over night at $4{ }^{\circ} \mathrm{C}$. The plates were washed $5 \times$ with PBS and blocked with $200 \mu \mathrm{l}$ RPMI 1640 (Gibco Invitrogen) containing $10 \%$ FCS for $30 \mathrm{~min}$ at room temperature. After coating with $100 \mu \mathrm{l}$ of cell sample $\left(10^{6}\right.$ cells $\left.\mathrm{ml}^{-1}\right)$ the plates were incubated for $24 \mathrm{~h}$ at $37^{\circ} \mathrm{C} / 5 \% \mathrm{CO}_{2}$. The plates were washed in PBS five times and $100 \mu \mathrm{l}$ anti-IgA-biotin $\left(1 \mu \mathrm{g} \mathrm{ml}^{-1}\right)$ in PBS $+0.5 \%$ FCS was added followed by $2 \mathrm{~h}$ incubation at room temperature. After $5 \times$ washing in PBS, $100 \mu \mathrm{l}$ per well of Streptavidin-HRP (diluted 1:500 in PBS $+0.5 \%$ FCS) was added followed by another $5 \times$ washing. Finally, $100 \mu \mathrm{l}$ of 3,3,5,5-tetramethylbenzidine substrate (Kem-En-Tec) was added to each well and plates were developed until distinct spots emerged followed by washing in deionized water and drying for $24 \mathrm{~h}$. Spots were counted using an automated ELISPOT Reader (AID, Strassberg, Germany).

MSD assays. In cytokine assays, cells were stimulated with $5 \mu \mathrm{g} \mathrm{ml}{ }^{-1}$ antigen and supernatants harvested after $72 \mathrm{~h}$ incubation at $37^{\circ} \mathrm{C} / 5 \%$ $\mathrm{CO}_{2}$. The levels of secreted cytokines were evaluated using Meso Scale Discovery assays. The Th1/Th2/Th17 cytokine 6-plex assay measuring IFN- $\gamma$, TNF- $\alpha$, IL-5, IL-10, IL-13, and IL-17 was performed using a MULTI-SPOT 96-well 7-Spot kit according to the manufacturer's instructions (Meso Scale Discovery, Gaithersburg, MD) and using a Sector Imager 2400 plate reader (Meso Scale Discovery).

Flow cytometric analysis. Cells were stimulated with $2 \mu \mathrm{g} \mathrm{ml}^{-1} \mathrm{ScpA}$ together with $1 \mu \mathrm{g} \mathrm{ml}^{-1}$ anti-CD28 (37.51; BD Biosciences) and anti-CD49d (9C10; BD Biosciences) for $1 \mathrm{~h}$ and subsequently incubated for $5 \mathrm{~h}$ at $37^{\circ} \mathrm{C}$ with $10 \mu \mathrm{g} \mathrm{ml}^{-1}$ brefeldin A (SigmaAldrich) in addition. After overnight storage at $4{ }^{\circ} \mathrm{C}$, cells were washed in FACS buffer (PBS containing $0.1 \%$ sodium azide and $1 \%$ FCS), and stained for $30 \mathrm{~min}$ at $4{ }^{\circ} \mathrm{C}$ (CXCR5: $60 \mathrm{~min}$ at room temperature) for surface markers with $\mathrm{mAbs}$ as indicated below. For intracellular staining, the cells were washed in FACS buffer, permeabilized using the Cytofix/Cytoperm kit (BD Biosciences), and stained for 30 min at $4{ }^{\circ} \mathrm{C}$ with $\mathrm{mAbs}$ as indicated below. Panel 1: anti-CD19-APC-eF780 (eBio1D3; eBioscience, San Diego, CA) anti-IgA-PE (mA-6E1; eBioscience). Panel 2: Surface; anti-CD4-APC-eF780 (RM4-5; eBioscience), anti-CD44-PE (IM7; BD Biosciences) and anti-CCR6AF647 (140706; BD Biosciences), intracellular; anti-IFN- $\gamma$-PE-Cy7 (XMG1.2; eBioscience, San Diego, CA) and anti-IL-17-PerCP-Cy5.5 (eBio17B7; eBioscience). Panel 3: anti-CD19-APC-eF780 (eBio1D3; eBioscience), anti-B220-PE-Cy7 (RA3-6B2; BD Biosciences), anti-IgD-PerCP-Cy5.5 (11-26c.2a; BD Biosciences), anti-CD95-APC (15A7; eBioscience) and anti-GL7-PE (GL7; BD Biosciences). Panel 4: Surface; anti-CD4-APC-eF780 (RM4-5; eBioscience),
anti-CD44-APC (IM7; BD Biosciences), anti-CXCR5-PE (BD Biosciences) and anti-PD1-PE-Cy7 (RMP1-30; BioLegend, San Diego, CA), intracellular; anti-IL-17-PerCP-Cy5.5 (eBio17B7; eBioscience). Panel 5: Surface; anti-CD4-APC-eF780 (RM4-5; eBioscience), antiCD44-APC (IM7; BD Biosciences), anti-CD62L-PE (MEL-14; BD Biosciences) and anti-CD69-PE-Cy7 (H1.2F3; BD Biosciences), intracellular; anti-IL-17-PerCP-Cy5.5 (eBio17B7; eBioscience). Cells were washed, resuspended in FACS buffer and analyzed using a FACSCanto flow cytometer (BD Biosciences). Responses were analyzed using FlowJo software v10.0.7. Doublets defined by FSC-A/FCS$\mathrm{H}$ were removed from datasets before analysis.

Statistical analysis. Immune responses were compared by one-way analysis of variance followed by Tukey's multiple comparison test of the means or unpaired $\mathrm{T}$ tests. A value of $P<0.05$ was considered significant. Statistical analyses were performed in GraphPad Prism v6.04 (GraphPad Software Inc.).

SUPPLEMENTARY MATERIAL is linked to the online version of the paper at http://www.nature.com/mi

\section{ACKNOWLEDGMENTS}

We thank The Danish Council for Independent Research, Technology and Production Sciences (Ref: 0602-01573B), EC-FP7: ADITEC (Grant agreement \#280873), and the Danish Strategic Research council (Centre for Nano Vaccines). Thanks to J. Rabech, R.F. Jensen and L. Rasmussen for excellent technical help and to J. Woodworth for critical reading of the manuscript.

\section{DISCLOSURE}

P.A. and I.R. are co-inventors on an issued patent (US8277823) covering the use of CAF01 as vaccine adjuvant. All rights have been assigned to Statens Serum Institut, a Danish not-for-profit governmental institute.

Official journal of the Society for Mucosal Immunology

\section{REFERENCES}

1. Agace, W.W. Tissue-tropic effector T cells: generation and targeting opportunities. Nat Rev Immunol 6, 682-692 (2006).

2. Turner, D.L. \& Farber, D.L. Mucosal resident memory CD4 T cells in protection and immunopathology. Front. Immunol 5, 331 (2014).

3. Thome, J.J. \& Farber, D.L. Emerging concepts in tissue-resident T cells: lessons from humans. Trends Immunol. 36, 428-435 (2015).

4. Jang, Y.S. et al. Lactoferrin causes IgA and IgG2b isotype switching through betaglycan binding and activation of canonical TGF-[beta] signaling. Mucosal Immunol 8, 906-917 (2014).

5. Karaffová, V. et al. Interaction of TGF- $\beta 4$ and IL-17 with IgA secretion in the intestine of chickens fed with E. faecium AL41 and challenged with S. Enteritidis. Res Vet Sci. 100, 75-79 (2015).

6. Roy, B., Brennecke, A.M., Agarwal, S., Krey, M., Duber, S. \& Weiss, S. An intrinsic propensity of murine peritoneal $\mathrm{B} 1 \mathrm{~b}$ cells to switch to $\lg \mathrm{A}$ in presence of TGF-beta and retinoic acid. PloS One 8, e82121 (2013).

7. Hou, S. et al. Follicular dendritic cell secreted protein FDC-SP controls IgA production. Mucosal Immunol 7, 948-957 (2014).

8. Hirota, K. et al. Plasticity of TH17 cells in Peyer's patches is responsible for the induction of T cell-dependent IgA responses. Nat. Immunol. 14, 372-379 (2013).

9. Way, E.E., Chen, K. \& Kolls, J.K. Dysregulation in lung immunity - the protective and pathologic Th17 response in infection. Eur. J. Immunol. 43, 3116-3124 (2013).

10. Wüthrich, M. et al. Vaccine-induced protection against 3 systemic mycoses endemic to North America requires Th17 cells in mice. J. Clin. Invest. 121, 554-568 (2011).

11. Jaffar, Z., Ferrini, M.E., Herritt, L.A. \& Roberts, K. Cutting edge: lung mucosal Th17-mediated responses induce polymeric lg receptor expression by the airway epithelium and elevate secretory IgA levels. J. Immunol. 182, 4507-4511 (2009). 
12. He, R. et al. Exaggerated IL-17 response to epicutaneous sensitization mediates airway inflammation in the absence of IL-4 and IL-13. J. Allergy. Clin. Immunol. 124, 761-770 e761 (2009).

13. Lindenstrom, T. et al. Tuberculosis subunit vaccination provides long-term protective immunity characterized by multifunctional CD4 memory T cells. J. Immunol. 182, 8047-8055 (2009).

14. Lindenstrom, T., Woodworth, J., Dietrich, J., Aagaard, C., Andersen, P. \& Agger, E.M. Vaccine-induced th17 cells are maintained long-term postvaccination as a distinct and phenotypically stable memory subset. Infect. Immun 80, 3533-3544 (2012).

15. Oleszycka, E. \& Lavelle, E.C. Immunomodulatory properties of the vaccine adjuvant alum. Curr. Opin. Immunol. 28, 1-5 (2014).

16. Bode, C., Zhao, G., Steinhagen, F., Kinjo, T. \& Klinman, D.M. CpG DNA as a vaccine adjuvant. Expert Rev. Vaccines 10, 499-511 (2011).

17. Anderson, K.G. et al. Intravascular staining for discrimination of vascular and tissue leukocytes. Nat. Protoc. 9, 209-222 (2014).

18. Jaffar, Z., Ferrini, M.E., Girtsman, T.A. \& Roberts, K. Antigen-specific Treg regulate Th17-mediated lung neutrophilic inflammation, B-cell recruitment and polymeric IgA and IgM levels in the airways. Eur. J. Immunol. 39, 3307-3314 (2009).

19. Khader, S.A. et al. IL-23 and IL-17 in the establishment of protective pulmonary $\mathrm{CD} 4+\mathrm{T}$ cell responses after vaccination and during Mycobacterium tuberculosis challenge. Nat. Immunol. 8, 369-377 (2007).

20. Cao, A.T., Yao, S., Gong, B., Elson, C.O. \& Cong, Y. Th17 cells upregulate polymeric Ig receptor and intestinal $\lg A$ and contribute to intestinal homeostasis. J. Immunol. 189, 4666-4673 (2012).

21. Cao, A.T., Yao, S., Gong, B., Nurieva, R.I., Elson, C.O. \& Cong, Y. Interleukin (IL)-21 promotes intestinal IgA response to microbiota. Mucosal Immunol 8, 1072-1082 (2015).

22. Pepper, M. et al. Different routes of bacterial infection induce long-lived TH1 memory cells and short-lived TH17 cells. Nat. Immunol 11, 83-89 (2010).

23. Trondsen, M. et al. Induction of local secretory IgA and multifunctional CD4(+) T-helper cells following intranasal immunization with a H5N1 whole inactivated influenza virus vaccine in BALB/c mice. Scand. J. Immunol. 81, 305-317 (2015).

24. Warfel, J.M. \& Merkel, T.J. Bordetella pertussis infection induces a mucosal $\mathrm{IL}-17$ response and long-lived Th17 and Th1 immune memory cells in nonhuman primates. Mucosal Immunol 6, 787-796 (2013).

25. Zielinski, C.E., Corti, D., Mele, F., Pinto, D., Lanzavecchia, A. \& Sallusto, F. Dissecting the human immunologic memory for pathogens. Immunol. Rev. 240, 40-51 (2011).

26. Shin, H. \& Iwasaki, A. Tissue-resident memory Tcells. Immunol. Rev. 255, 165-181 (2013).

27. Turner, D.L. et al. Lung niches for the generation and maintenance of tissue-resident memory T cells. Mucosal Immunol 7, 501-510 (2014).
28. Teijaro, J.R., Turner, D., Pham, Q., Wherry, E.J., Lefrancois, L. \& Farber, D.L. Cutting edge: Tissue-retentive lung memory CD4 T cells mediate optimal protection to respiratory virus infection. J. Immunol. 187, 5510-5514 (2011).

29. Sakai, S. et al. Cutting edge: control of Mycobacterium tuberculosis infection by a subset of lung parenchyma-homing CD4 Tcells. J. Immunol. 192, 2965-2969 (2014).

30. Clark, R.A. et al. The vast majority of CLA + T cells are resident in normal skin. J. Immunol. 176, 4431-4439 (2006).

31. lijima, N. \& Iwasaki, A. T cell memory. A local macrophage chemokine network sustains protective tissue-resident memory CD4 T cells. Science 346, 93-98 (2014).

32. Shinoda, K. et al. Type II membrane protein CD69 regulates the formation of resting T-helper memory. Proc. Natl. Acad. Sci. U.S.A. 109, 7409-7414 (2012).

33. Bedoya, S.K., Lam, B., Lau, K. \& Larkin, J. Th17 Cells in Immunity and Autoimmunity. Clin. Dev. Immunol. 2013, 16 (2013).

34. Schmitt, N. \& Ueno, H. Regulation of human helper $T$ cell subset differentiation by cytokines. Curr. Opin. Immunol. 34, 130-136 (2015).

35. Lorenzen, E. et al. Intramuscular priming and intranasal boosting induce strong genital immunity through secretory $\lg A$ in minipigs infected with Chlamydia trachomatis. Front. Immunol 6, 628 (2015).

36. Cleary, P.P., Matsuka, Y.V., Huynh, T., Lam, H. \& Olmsted, S.B. Immunization with $\mathrm{C} 5 \mathrm{a}$ peptidase from either group A or B streptococci enhances clearance of group A streptococci from intranasally infected mice. Vaccine 22, 4332-4341 (2004).

37. Aagaard, C. et al. A multistage tuberculosis vaccine that confers efficient protection before and after exposure. Nat. Med 17, 189-194 (2011).

38. Davidsen, J. et al. Characterization of cationic liposomes based on dimethyldioctadecylammonium and synthetic cord factor from M. tuberculosis (trehalose 6,6'-dibehenate)-a novel adjuvant inducing both strong $\mathrm{CMl}$ and antibody responses. Biochim. Biophys. Acta. 1718, 22-31 (2005).

(c)(1)(2) This work is licensed under a Creative Commons (1) Attribution-NonCommercial-ShareAlike 4.0 International License. The images or other third party material in this article are included in the article's Creative Commons license, unlessindicated otherwise in the creditline; if thematerial is not included under the Creative Commons license, users will need to obtain permission from the license holder to reproduce the material. To view a copy of this license, visit http:// creativecommons.org/licenses/by-nc-sa/4.0/ 